\title{
Um Framework para Desenvolvimento de Sistemas de Gerenciamento de Informações de Desastres Naturais
}

\author{
Carlos Henrique Tavares Brumatti, Erick Lima Figueiredo, \\ Lucas Fouraux Dorigueto, Jugurta Lisboa-Filho
}

Departamento de Informática - Universidade Federal de Viçosa - Viçosa - MG - Brasil

\{carlos.h.tavares, erick.figueiredo, lucas.dorigueto, jugurta\}@ufv.br

\begin{abstract}
Disaster Information Management Systems (DIMS) are systems of most importance for public management and can be used before, during and after emergency situations. Associating this type of system with Voluntary Geographic Information Systems (VGI) represents a major advance in the relationship of society with its managers. Thus, this work presents a framework for the development of DIMS using VGI, seeking to follow patterns of data sharing and usability.
\end{abstract}

Resumo. Sistemas de Gerenciamento de Informação de Desastres (DIMS) são sistemas de extrema importância para a gestão pública, podendo ser utilizados antes, durante e após situações de emergência. Associar esse tipo de sistema com Informação Geográfica Voluntária (VGI) representa um grande avanço no que diz respeito ao relacionamento da sociedade com os seus gestores. Dessa forma, esse trabalho apresenta um framework para o desenvolvimento de DIMS utilizando VGI, buscando garantir interoperabilidade por meio da adoção de padrões de compartilhamento de dados e de usabilidade.

\section{Introdução e Fundamentação Teórica}

Os impactos da ocorrência de desastres naturais vão muito além dos danos materiais. Quando um evento desses ocorre, bens e serviços deixam de ser produzidos e ofertados em um dado intervalo de tempo, impactando assim diretamente na economia local. Além disso, as áreas afetadas ficam estruturalmente depreciadas e a população local passa a necessitar do amparo do poder público de maneira rápida e emergencial (PAHO 2008).

Nesse sentido, Sistemas de Gerenciamento de Informações de Desastres (DIMS) podem ser utilizados por gestores de órgãos de atendimento à ocorrência de desastres, a fim de facilitar no processo de tomada de decisão. Um obstáculo para isso, no entanto, acaba sendo a falta de padronização nos dados do próprio DIMS, conforme dito por Ryoo e Choi (2006), o que atrapalha a interoperabilidade entre os diversos Sistemas de Informação Geográfica (SIG) disponíveis. No contexto atual de informatização do setor público, sistemas de software open source, como o QGIS por exemplo, são amplamente utilizados, sobretudo no processo de gestão territorial dos municípios, alavancando assim a produção de mapas temáticos e de quaisquer outras informações espaciais referentes ao município, que quando utilizadas em conjunto auxiliam como fonte de informação para o processo de tomada de decisão.

Goodchild (2007) batizou uma nova forma de obtenção de informação espacial, que é feita por meio de Sistemas de Informação Geográfica Voluntária (VGI). Ou seja, 
os "usuários sensores" ou "producers" colaboram por meio de sistemas Web 2.0 de forma voluntária com alguma informação acerca da sua realidade, normalmente apoiadas a imagens obtidas por meio de sensoriamento remoto. Uma das vantagens desse tipo de aplicação é que o usuário não precisa passar por nenhum treinamento muito específico sobre como produzir a informação espacial, basta aprender a usar o sistema.

Dessa forma, o objetivo deste projeto é desenvolver um framework para facilitar a implementação, em situações de emergência, de DIMS utilizando VGI, buscando garantir interoperabilidade por meio da adoção de padrões de compartilhamento de dados e de usabilidade.

\section{Metodologia de Pesquisa}

Um framework pode ser definido como sendo um conjunto básico de código de programação que pode ser configurado ou editado para alguma finalidade específica. Neste caso, a ferramenta é voltada para o desenvolvimento rápido de DIMS que utilizam VGI para obtenção dos dados. A arquitetura geral do framework pode ser vista na Figura 1.

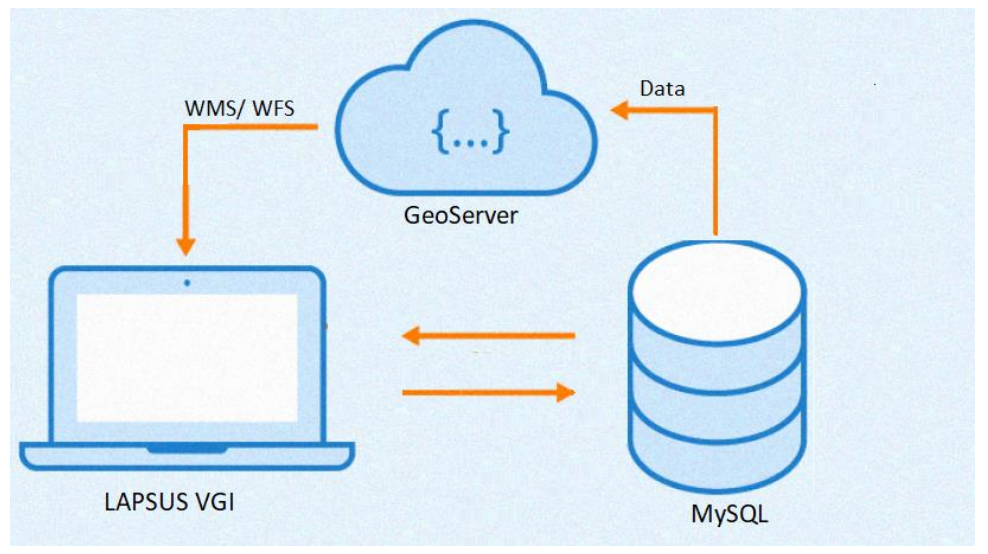

Figura 1: Arquitetura geral do framework. Fonte: Autores

O framework LapsusVGI (Dorigueto 2020) foi concebido de forma a seguir as recomendações das normas ISO 22327 (ISO 2018) e ISO 22351 (ISO 2015) em relação à implementação da estrutura dos mapas do sistema, dos ícones de emergência, da modelagem conceitual do banco de dados e da exportação das colaborações feitas no formato eXtensible Markup Language (XML). Além disso, o sistema GeoServer fornece os dados espaciais nos padrões Web Map Feature (WMS) e Web Feature Service (WFS), conforme as especificações do Open Geospatial Consortium (OGC) (OGC 2021).

Todas as interfaces do sistema foram desenvolvidas utilizando HTML, CSS e Bootstrap, buscando-se desenvolver telas que fossem as mais responsivas possíveis. Atrelado a isso, usou-se a biblioteca gráfica Leaflet como provedora dos mapas do sistema. O banco de dados escolhido para a persistência dos dados foi o MySQL, sendo a linguagem PHP responsável por fazer a conexão de todo o sistema com ele. Todo o código foi disposto seguindo a arquitetura Model-View-Controller (MVC), facilitando assim o processo de manutenção e adição de novas funcionalidades no sistema. 


\section{Resultados}

A configuração de um novo sistema que utiliza o framework aqui descrito é realizada pelo usuário administrador. Após realizar o login, é apresentado para esse tipo de usuário o menu com as opções, e dentre elas há a "Configuração". Após selecionada, é apresentado um formulário com vários campos, destacando-se o nome que o sistema vai apresentar e o mapa para seleção da região de interesse.

Já no processo de colaboração, o usuário colaborador associa seus dados a um dos tipos de dados vetoriais existentes, isto é, ponto, linha ou polígono, buscando assim dimensionar a informação colaborada. Uma vez que a informação está no sistema, a colaboração passa por um processo de validação pelo usuário moderador, que aceita ou rejeita uma colaboração realizada por um usuário registrado ou anônimo. Uma vez aprovado, a colaboração fica disponível tanto para visualização quanto para a exportação no formato XML, permitindo assim a interoperabilidade dessa ferramenta com outros sistemas (ex.: SIG ou DIMS). Além disso, há como importar dados espaciais no formato shapefile para o sistema, a fim de oferecer informações adicionais para o usuário colaborador. A Figura 2 mostra a tela de colaboração do sistema quando acessada de maneira anônima.

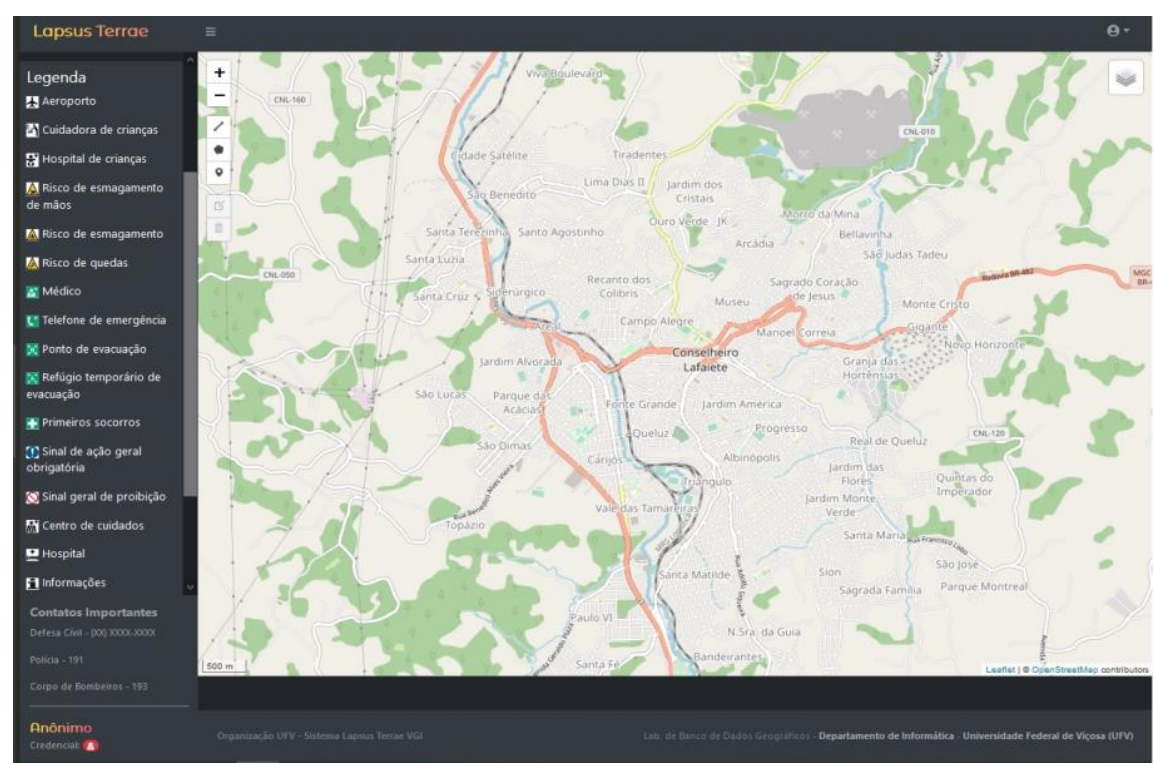

Figura 2: Tela de contribuição do usuário anônimo. Fonte: Autores

No lado esquerdo da tela de colaboração, localiza-se a legenda do mapa, com ícones que estarão dispostos no mapa conforme a seleção do atributo pelo usuário no menu de agrupamento de camadas. Esse menu é alimentado dinamicamente conforme o sistema é configurado com arquivos geojson, indicando a localização das estruturas. No mapa de colaboração, tem-se como pano de fundo camadas do OpenStreetMap, com possibilidade de alteração para uma imagem de satélite da região. As geometrias das colaborações também ficam dispostas nesse mesmo tipo de mapa, com os dados das colaborações exibidos em um menu popup conforme o clique do usuário.

\section{Conclusão}

Este trabalho aborda o desenvolvimento de um framework open source focado no desenvolvimento de DIMS que utilizam VGI como forma de obtenção de dados, 
seguindo padrões internacionais de interoperabilidade e gerenciamento de sistemas de emergência.

Como trabalhos futuros, pretende-se transcrever todo o código para NodeJS, reescrevendo o frontend da aplicação com a framework Vue.js, permitindo a reutilização de código por meio do uso de componentes. Em relação ao backend, pretende-se desenvolver uma Interface de Programação de Aplicativos (API) para o fornecimento de dados, permitindo assim que todos os serviços possam ser disponibilizados por meio de rotas, facilitando o desenvolvimento de novos produtos. $\mathrm{O}$ projeto utiliza somente tecnologias open source, e pode ser encontrado no endereço: http://www.dpi.ufv.br/projetos/lapsusVGI/.

\section{Agradecimentos}

Projeto parcialmente financiado com recursos da Fapemig. Os autores receberam bolsa e apoio da CAPES, CNPq e UFV.

\section{Referências}

Dorigueto, Lucas F., Brumatti, Carlos H. T., Lisboa-Filho, Jugurta (2020) LapsusVGI: um framework para sistemas de gerenciamento de informação sobre deslizamento de terra. In: Simpósio Brasileiro de Geoinformática (GEOINFO), 21, São José dos Campos. p. 264-269.

Goodchild, M. F. (2007) Citizens as sensors: the world of volunteered geography. GeoJournal,69(4): 211-221.

ISO - International Organization for Standardization (2018). ISO 22327:2018. Security and resilience - Emergency management - Guidelines for implementation of a community-based landslide early warning system.

ISO - International Organization for Standardization (2015). ISO/TR 22351:2015. Societal security - Emergency management - Message structure for exchange of information.

OpenStreetMap (2021) Disponível em: https://www.openstreetmap.org/about. Acessado em: 16/03/2021.

OGC - Open Geospatial Consortium. (2020) Web Map Service. Disponível em: https://www.ogc.org/standards/wms.

Pan American Health Organization - PAHO (2008) Manual para avaliação do impacto socioeconômico e ambiental dos desastres. Curso Internacional para Gerentes sobre Saúde, Desastres e Desenvolvimento. Disponível em: http://www.disasterinfo.net/lideres/portugues/04/pdfs/avaliacao_de_impacto.pdf. Acessado em: 02/03/2021.

Ryoo, J., Choi, Y. B. (2006) A comparison and classification framework for disaster information management systems. International Journal of Emergency Management, 3: 264-279 